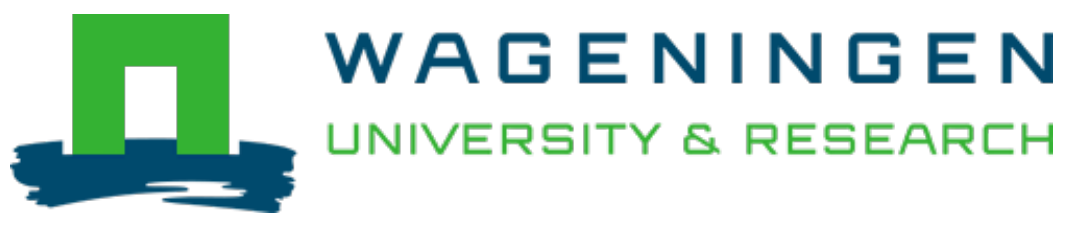

\title{
Engineering the Protein Corona Structure on Gold Nanoclusters Enables Red- Shifted Emissions in the Second Near-infrared Window for Gastrointestinal Imaging
}

\author{
Angewandte Chemie-International Edition \\ Wang, Weili; Kong, Yifei; Jiang, Jun; Xie, Qainqian; Huang, Yang et al \\ https://doi.org/10.1002/anie.202010089
}

This publication is made publicly available in the institutional repository of Wageningen University and Research, under the terms of article $25 \mathrm{fa}$ of the Dutch Copyright Act, also known as the Amendment Taverne. This has been done with explicit consent by the author.

Article 25 fa states that the author of a short scientific work funded either wholly or partially by Dutch public funds is entitled to make that work publicly available for no consideration following a reasonable period of time after the work was first published, provided that clear reference is made to the source of the first publication of the work.

This publication is distributed under The Association of Universities in the Netherlands (VSNU) 'Article $25 \mathrm{fa}$ implementation' project. In this project research outputs of researchers employed by Dutch Universities that comply with the legal requirements of Article 25fa of the Dutch Copyright Act are distributed online and free of cost or other barriers in institutional repositories. Research outputs are distributed six months after their first online publication in the original published version and with proper attribution to the source of the original publication.

You are permitted to download and use the publication for personal purposes. All rights remain with the author(s) and / or copyright owner(s) of this work. Any use of the publication or parts of it other than authorised under article $25 \mathrm{fa}$ of the Dutch Copyright act is prohibited. Wageningen University \& Research and the author(s) of this publication shall not be held responsible or liable for any damages resulting from your (re)use of this publication.

For questions regarding the public availability of this publication please contact openscience.library@wur.nl 


\title{
Engineering the Protein Corona Structure on Gold Nanoclusters Enables Red-Shifted Emissions in the Second Near-infrared Window for Gastrointestinal Imaging
}

\author{
Weili Wang ${ }^{+}$, Yifei Kong ${ }^{+}$Jun Jiang, Qianqian Xie, Yang Huang, Guanna Li, Di Wu, \\ Huizhen Zheng, Meng Gao, Shujuan Xu, Yanxia Pan, Wei Li, Ronglin Ma, Mei X. Wu, \\ Xuehua Li, Han Zuilhof, Xiaoming Cai, and Ruibin Li*
}

\begin{abstract}
The application of NIR-II emitters for gastrointestinal (GI) tract imaging remains challenging due to fluorescence quenching in the digestive microenvironment. Herein, we report that red-shifting of the fluorescence emission of $\mathrm{Au}$ nanoclusters (AuNCs) into NIR-II region with improved quantum yields $(Q Y)$ could be achieved by engineering a protein corona structure consisting of a ribonuclease- $A$ (RNase-A) on the particle surfaces. RNase-A-encapsulated AuNCs (RNase-A@AuNCs) displayed emissions at $1050 \mathrm{~nm}$ with a $1.9 \% Q Y$. Compared to rare earth and silver-based NIR-II emitters, RNase-A@AuNCs had excellent biocompatibility, showing $>50$-fold higher sensitivity in GI tract, and migrated homogenously during gastrointestinal peristalsis to allow visualization of the detailed structures of the GI tract. RNase-A@AuNCs could successfully examine intestinal tumor mice from healthy mice, indicating a potential utility for early diagnosis of intestinal tumors.
\end{abstract}

[*] Dr. W. Wang, ${ }^{[+]}$J. Jiang, Q. Xie, D. Wu, H. Zheng, M. Gao, S. Xu, Y. Pan, W. Li, R. Ma, Prof. R. Li

State Key Laboratory of Radiation Medicine and Protection, School for Radiological and Interdisciplinary Sciences (RAD-X), Collaborative Innovation Center of Radiological Medicine of Jiangsu Higher Education Institutions, Soochow University

Suzhou, 215123, Jiangsu (China)

E-mail: liruibin@suda.edu.cn

Dr. Y. Kong ${ }^{[+]} \mathrm{M}$. X. Wu

Wellman Center for Photomedicine, Massachusetts General Hospital, Harvard Medical School, Boston, MA 02114 (USA)

Y. Huang, X. Li

Key Laboratory of Industrial Ecology and Environmental Engineering, School of Environmental Science and Technology, Dalian University of Technology, Dalian, 116024 (China)

G. Li, H. Zuilhof

Laboratory of Organic Chemistry, Wageningen University

Stippeneng 4, 6703 WE, Wageningen (The Netherlands) and

Department of Chemical and Materials Engineering, Faculty of Engineering, King Abdulaziz University, Jeddah (Saudi Arabia)

X. Cai

School of Public Health, Soochow University, Suzhou, 215123, Jiangsu (China)

$\left.{ }^{+}\right]$These authors contributed equally to this work.

(2) Supporting information and the ORCID identification number(s) for (D) the author(s) of this article can be found under:

https://doi.org/10.1002/anie.202010089.

\section{Introduction}

Barium swallow and endoscopic techniques are the mainstream techniques for gastrointestinal (GI) imaging. ${ }^{[1]}$ However, barium swallow is an X-ray based diagnosis technique with limited spatial resolution and notable radiation risk; endoscopy is an invasive diagnostic technique contraindicated in patients with suspected bowel stricture or obstruction. Therefore, non-invasive and X-ray-free imaging technologies are highly desired for early diagnosis of some severe GI diseases, especially tumors. ${ }^{[2]}$

Fluorescence emissions in the second near infrared window (NIR-II, 1000-1400 nm) have attracted substantial research enthusiasms for bio-imaging. ${ }^{[3]}$ Since the excitation and emission wavelengths involved in obtaining NIR-II emissions already allow deep tissue penetration as display a strongly reduced autofluorescence and photon scattering, one-photon excitation was often used as laser source in NIRII imaging. Compared to small molecular NIR-II emitters (e.g. IR1061, ${ }^{[4]} \mathrm{CH} 1055^{[5]}$ and BTC1070 ${ }^{[6]}$ ), nano-sized NIR-II emitters including quantum dots (QDs) ${ }^{[7]}$ rare earth nanoparticle (RENP) ${ }^{[8]}$ and single wall carbon nanotube (SWNT) ${ }^{[9]}$ show relatively higher quantum yields (QYs), and lower susceptibility to photobleaching. They have been exploited for liver, ${ }^{[10]}$ kidney, $^{[11]}$ brain $^{[5]}$ and lung ${ }^{[12]}$ imaging. However, few reports focus on NIR-II imaging of GI tract. As the acidic and enzymatic bio-contexts of GI tract may lead to fluorescence quenching of most nano-sized emitters, we proposed to synthesize inert metal (e.g. Au, Pt) based emitters for NIR-II imaging of GI tract. ${ }^{[13]}$

In this study, we exploited a theory of ligand-metal-metal charge transfer (LMMCT) ${ }^{[14]}$ to synthesize Au-based nanoclusters (AuNCs) with emissions in NIR-II region. Ribonuclease-A (RNase-A) were therefore selected to encapsulate gold nanocluster (RNase-A@AuNC) for red-shifted emissions as it consists of a desired cocktail of thiol group (cysteine) and aromatic amino acids (histidine and tyrosine). The resulting RNase-A@AuNCs were exposed to GI tract simulated fluids and mammalian cells for stability and biosafety assessments. The in vivo imaging capability of RNase-A@AuNC in GI tract was examined in mice by oral administration. Finally, we employed an intestinal cancer model to justify the potential utility of AuNCs as an imaging agent for tumor diagnosis. 


\section{Results and Discussion}

Considering the biocompatibility and diversity of amino acids, we examined the feasibility of constructing aromatic amino acids on particle surfaces to reduce the fermi energy of AuNCs. A density functional theory (DFT) computations were performed to investigate the band energy between the highest occupied molecular orbital (HOMO) and lowest unoccupied molecular orbital (LUMO) of AuNCs. AuNCs binding with cysteine (Cys), histidine (His) and tyrosine (Tyr) showed band gaps at $2.308 \mathrm{eV}, 0.038 \mathrm{eV}$ and $0.104 \mathrm{eV}$, respectively (Figure $1 \mathrm{~A})$. These three amino acids were exploited to synthesize AuNCs by a microwave assisted method, ${ }^{[15]}$ including $\mathrm{Au}^{3+}$ binding with ligands, reduction by $\mathrm{NaBH}_{4}$ and nucleation to form clusters. The emission wavelength and morphologies were examined to assess the impacts of Cys, His and Tyr (Table S1). While Cys could greatly facilitate the formation of Au clusters, introduction of His and Tyr was able to induce redshifts of the emission wavelengths. However, the resulting AuNCs had low quantum yields (QYs) probably due to low surface coverage.

We attempted to control the coverage of Cys, His and Tyr on particle surfaces by constructing a corona structure of RNase-A, which consists of desired number of Cys (8), His (4) and Tyr (6). For a comparison, a ligand library of glutathione, mercaptoethanol, dihydrolipoic acid, lipoic acid-based sulfobetaine (LA-sulfobetaine), and bovine serum albumin (BSA) was also included (Table S2). These ligands consist of different numbers of soft (HS-) and hard groups (e.g. Tyr and His) as well as charges, which may significantly impact the emissions of AuNCs based on LMMCT theory. Figure 1B depicted an illustration proposing the reaction path for synthesizing RNase-A@AuNCs. The globular RNase-A proteins were fully unfolded to form loose and expanded random-coil chains through cleavage of disulfide bonds by redox agents under strong basic conditions (Figure S1).
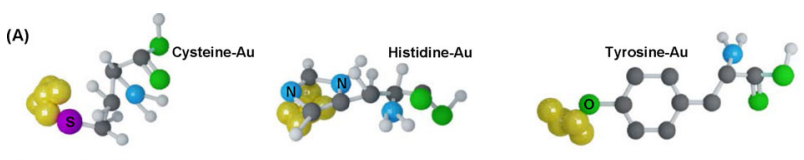

Molecular orbitals
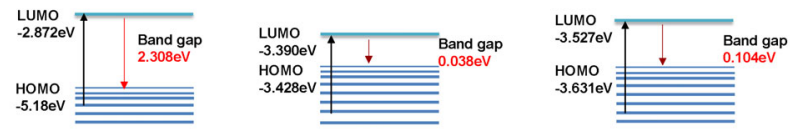

(B)

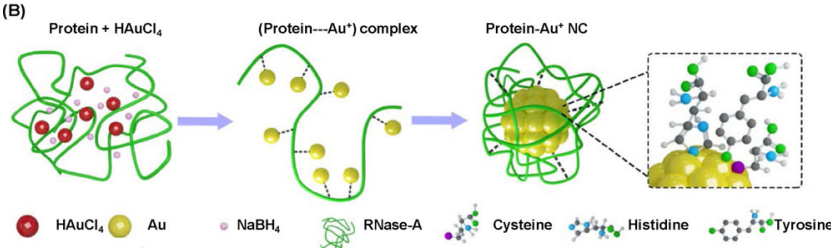

Figure 1. Band gaps of Cys/His/Tyr-Au composites and synthesis route of RNase-A@AuNCs. A) Band gaps of Cys-, His-, and Tyr-Au composites by DFT calculations. B) Schematic representation of the reaction path for the synthesis of RNase-A@AuNCs. The 3D arrangement of RNase-A dissociates in basic and reductive conditions. After nucleation of $\mathrm{Au}$ atoms under microwave irradiation, the dissociated peptide chains coordinate with $\mathrm{Au}$ atoms to facilitate the maturation of $\mathrm{Au}$ clusters.
Thereupon, the exposed functional groups (Cys, His and Tyr) along the peptide chains will coordinate with Au cations to assist the formation of AuNCs under microwave irradiation. The interaction of RNase-A with gold nanoclusters was further investigated by molecular dynamics simulation (MD). As shown in Figure $\mathrm{S} 2$, the $\mathrm{Au}_{25} \mathrm{NCs}$ were stabilized by forming RNase-A@ $\mathrm{Au}_{25} \mathrm{NC}$ complexes at specific binding sites. In all three tested model, Cys, Tyr and His were found to bind on AuNC surface.

As expected, RNase-A encapsulated AuNCs were found to display a perfect Gaussian-shaped emission peak centered at $1050 \mathrm{~nm}$ with a full width at half-maximum (FWHM) of $\approx 205 \mathrm{~nm}$ (Figure 2 A), which is relatively narrow compared to most reported novel-metal based imaging agents. The emission spectra of RNase-A@AuNCs had a nice overlap with the NIR transparency window of tissues (800$1400 \mathrm{~nm}) .^{[3]}$ The morphology and primary size of RNaseA@AuNCs were examined by transmission electron microscopy (TEM). As shown in Figure 2B, RNase-A@AuNCs had an average diameter of $2.2 \pm 0.1 \mathrm{~nm}$ and homogenously distributed on copper mesh grids. The corresponding selected area electron diffraction (SAED) pattern in the diffraction rings was indicative of AuNCs that were randomly oriented and had face centered cubic Au structure (Figure 2C). The individual particles were further examined by high-resolution (HR) TEM and displayed a high crystalline structure with an

(A)

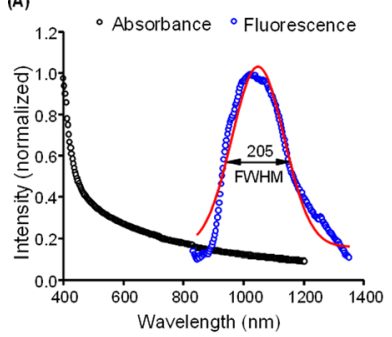

(B)

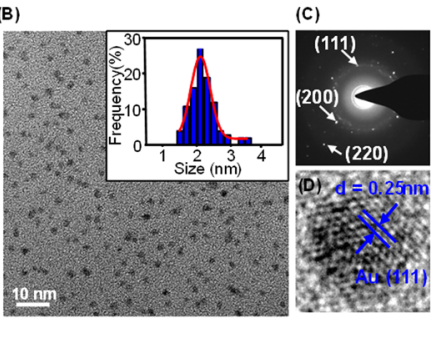

(E)

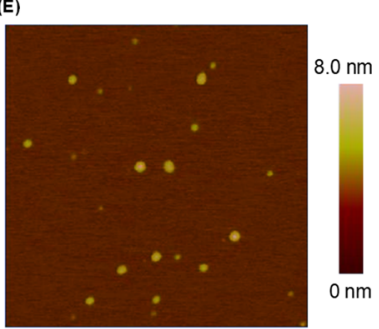

Figure 2. A) UV/Vis/NIR absorption and photoluminescence spectra of the RNase-A@AuNCs.2 mg mL ${ }^{-1}$ RNase-A@AuNCs were dissolved in water to examine their UV/Vis/NIR absorption by UV/Vis-NIR spectrophotometry. The photoluminescence spectra were acquired by fluorescence spectrophotometry, the solid red line is the Gaussian function fit from Origin Lab 8.0. B) TEM image, C) selected area electron diffraction (SAED) pattern, D) HR-TEM image, and E) AFM image of the assynthesized RNase-A@AuNCs. The RNase-A@AuNCs at $2 \mathrm{mg} \mathrm{mL}^{-1}$ were dispersed in water and dropped in Cu-grids or mica for dryness at room temperature. The inset of the TEM image shows size histogram of 100 particles. F) Plot of the integrated fluorescence spectra of both RNase-A@AuNCs and IR-26. A reference near-infrared fluorophore (IR-26) at five different concentrations was used to calculate the quantum yield of RNase-A@AuNCs by comparing the slopes of the linear fits. 
inter-planar spacing of $0.25 \mathrm{~nm}$ (Figure 2D). Since RNase-A proteins are transparent under TEM, atomic-force microscopy (AFM) was exploited to assess the overall dimension of RNase-A@AuNCs at $3.5 \pm 0.5 \mathrm{~nm}$ (Figure 2E). The thickness of corona structure on Au cluster was at $0.65 \mathrm{~nm}$ determined by equation 1 (supplementary methods). The quantum yield (QY) of RNase-A@AuNCs was 1.9\% determined by comparison to a reference material (IR 26) with reported QY of $0.5 \%^{[16]}$ (Figure $2 \mathrm{~F}$ ). The QY of RNase-A@AuNCs was significantly higher than the values $(0.012-0.6 \%)$ of most reported NIR-II emitting molecules. ${ }^{[17]}$

Three subgroups of $\mathrm{Au}$ clusters $\left(\mathrm{Au}_{17}, \mathrm{Au}_{20}\right.$ and $\left.\mathrm{Au}_{25}\right)$ could be detected in the as-prepared RNase-A@AuNCs by matrix-assisted laser desorption ionization time-of-flight (MALDI-TOF) mass spectrometry (Figure S3). Proteomic analysis was performed to examine the number of Cys, His and Tyr binding on AuNC surfaces (Figure S4, Table S3). As a result, each AuNC particle could bind with an average of 17 amino acids, including $8 \mathrm{Cys}, 4$ His and 5 Tyr. Since $\mathrm{Au}_{17}$ and $\mathrm{Au}_{20}$ theoretically bind less amino acids than $\mathrm{Au}_{25}$, we speculated that $\mathrm{Au}_{25}$ might bind all Cys, His and Tyr of a RNase-A molecule and the number of amino acids binding on $\mathrm{Au}_{25}$ could come up to 18. This number is consistent with Zheng et al. report that $\mathrm{Au}_{25}$ provides 18 binding sites on the surface. ${ }^{[18]}$ The purity of RNase-A@AuNC was examined by gel separation. RNase-A@AuNCs merely displayed one band around the theoretical molecular weight of the prominent constitute (RNase-A@Au25) at $\approx 18.6 \mathrm{KD}$. RNaseA@AuNCs showed a significant migration shift compared to RNase-A proteins, suggesting a successful attachment of RNase-A on AuNC surfaces (Figure S5). The interactions of AuNCs with three animo acids could be evidenced by the amide II band of Fourier transform infrared (FTIR) spectra (Figure S6).

Since GI tract consists of acidic and enzymatic biocontexts, it may digest NIR-II emitters to affect their optical activities and elicit hazard effects. ${ }^{[19]}$ To assess the stability in GI tract, RNase-A@AuNCs were exposed to a simulated gastric juice (SGJ) ${ }^{[20]}$ and PBS buffer to examine fluorescence changes and compared with two reported NIR-II nanoemitters including $\mathrm{Ag}_{2} \mathrm{~S}$ and $\mathrm{NaYF}_{4}: \mathrm{Er} / \mathrm{Yb}$ (Figure $3 \mathrm{~A}$ ). As expected, RNase-A@AuNCs were robust to the acidic and enzymatic digestion process. While RNase-A@AuNCs showed only $25 \%$ decreases of fluorescence in $2 \mathrm{~h}$, other emitters displayed more than $95 \%$ quenching effect in SGJ. The morphology and primary size of RNase-A@AuNCs, $\mathrm{Ag}_{2} \mathrm{~S}$ and $\mathrm{NaYF}_{4}: \mathrm{Er} / \mathrm{Yb}$ in SGJ were examined by TEM. $\mathrm{Ag}_{2} \mathrm{~S}$ and $\mathrm{NaYF}_{4}: \mathrm{Er} / \mathrm{Yb}$ underwent significant biotransformation in SGJ, evidenced by particle morphology and hydrodynamic size changes (Figure S7 and S8). Furthermore, the biosafety of RNase-A@AuNCs was assessed in two intestine epithelial cells (Caco-2, HCT116). As shown in Figure 3B, RNaseA@AuNCs showed extraordinary biocompatibility in two cell lines in a wide range of exposure doses $\left(0-500 \mu \mathrm{g} \mathrm{mL}^{-1}\right)$, whereas $\mathrm{NaYF}_{4}: \mathrm{Er} / \mathrm{Yb}$ and $\mathrm{Ag}_{2} \mathrm{~S}$ induced dose-dependent cytotoxicity. Furthermore, we used a LIVE/DEAD staining assay to visualize cell viability by confocal microscopy. As a result, substantial dead cells could be detected in two cell lines exposed to $\mathrm{NaYF}_{4}: \mathrm{Er} / \mathrm{Yb}$, displaying massive red
(A)
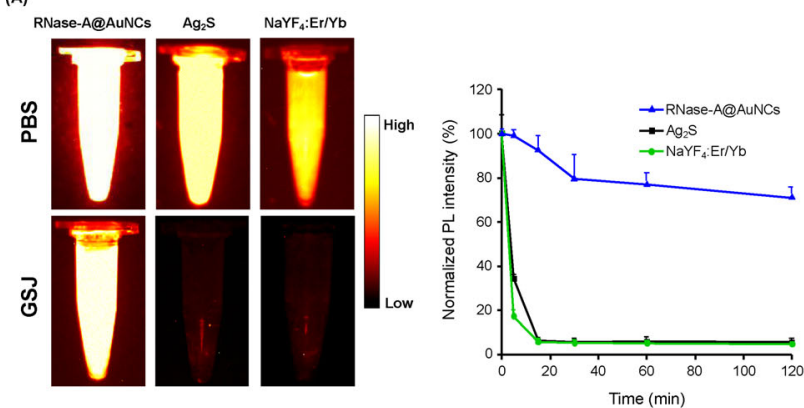

(B)
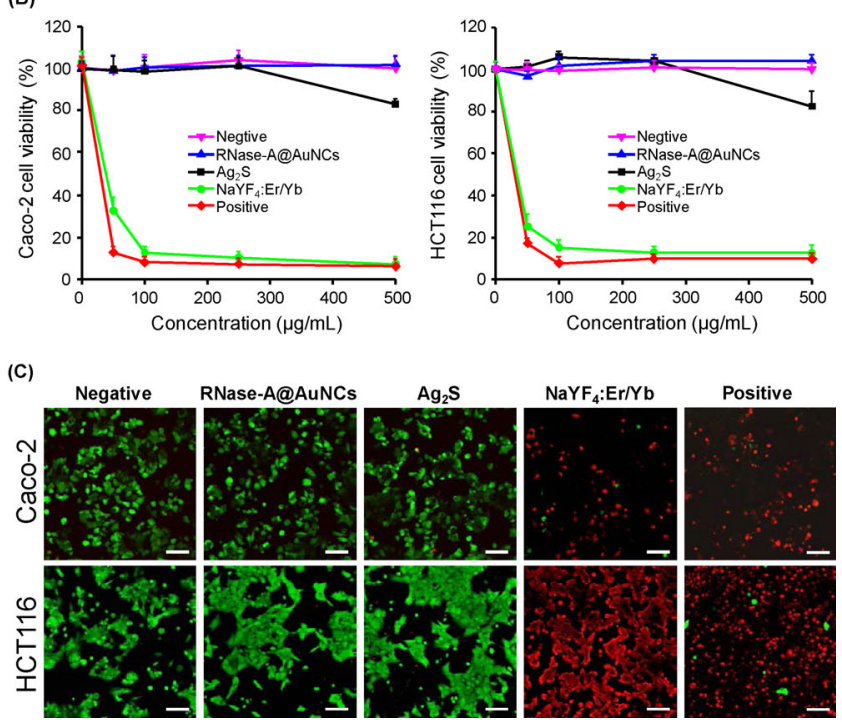

Figure 3. A) Assessment of fluorescence stability of nanosized NIR-II emitters in SG) and PBS buffer. $\mathrm{Ag}_{2} \mathrm{~S}, \mathrm{NaYF}_{4}: \mathrm{Er} / \mathrm{Yb}$, and RNaseA@AuNCs were dissolved in $\mathrm{DI} \mathrm{H}_{2} \mathrm{O}$ or SG) at $2 \mathrm{mg} \mathrm{mL}^{-1}(n=3)$. Data are presented as mean values \pm SD. Calculation of PL is described in supplementary methods. B) Cell viability test. Caco-2 and HCT116 cells were exposed to particles at concentrations of 0 $500 \mu \mathrm{g} \mathrm{mL}^{-1}$ for $24 \mathrm{~h}$. Cell viabilities were assessed by MTS assay ( $n=3$ biologically independent cell samples). Data are presented as mean values \pm SD. C) Fluorescence microscopy images by LIVE/ DEAD staining. The cells were exposed to $500 \mu \mathrm{g} \mathrm{mL}^{-1}$ particles for $24 \mathrm{~h}$. Then the cells were stained by a LIVE/DEAD staining kit for microscopy imaging. Scale bars $=100 \mu \mathrm{m}$.

fluorescence of Ethidium homodimer-1 (EthD-1) binding with DNA (Figure 3C). This is probably because cellular internalization of RNase-A@AuNCswere limited except for a few associations with membranes (Figure S9).

The biokinetics of oral injected RNase-A@AuNC is determined by inductively coupled plasma-optical emission spectrometry (ICP-OES) in different organs at $24 \mathrm{~h}$ post administration. The ICP-OES-based quantitative analysis indicated that fecal excretion $(\approx 91 \%)$ was the predominant pathway for the clearance of the injected nanoparticles, and $7 \%$ AuNCs were found in stomachs (Figure S10). The hematoxylin and eosin (H\&E) staining images demonstrated that this AuNC probe had negligible toxicity to GI tract, heart, pancreas, liver and kidney (Figure S11). These results indicated that RNase-A@AuNCs had excellent biocompatibility and well sustained their NIR-II emission in GI tract microenvironment due to their negative surface charge and 
high stability, denying cellular internalization as well as biotransformation-induced hazard effects. ${ }^{[21]}$

Before tumor imaging, RNase-A@AuNCs were orally injected into healthy C57BL/6 mice to examine signal-dose relationships in GI tract, and compared with $\mathrm{Ag}_{2} \mathrm{~S}$ and $\mathrm{NaYF}_{4}: \mathrm{Er} / \mathrm{Yb}$. As shown in Figure $4 \mathrm{~A}$, the fluorescence emissions of RNase-A@AuNCs got to a plateau at an administration dose of $10 \mathrm{mg} \mathrm{kg}^{-1}$. The sensitivity of each emitter was determined by the slope of signal-dose curve. RNase-A@AuNCs displayed >50 higher sensitivity than $\mathrm{Ag}_{2} \mathrm{~S}$ and $\mathrm{NaYF}_{4}: \mathrm{Er} / \mathrm{Yb}$. A dynamic movement process of AuNCs was observed in gastrointestinal peristalsis, including stomach duodenum, ileum and caecum (Figure 4B). Fluorescence intensity analysis of regions of interests (ROIs) ${ }^{[16]}$

(A)
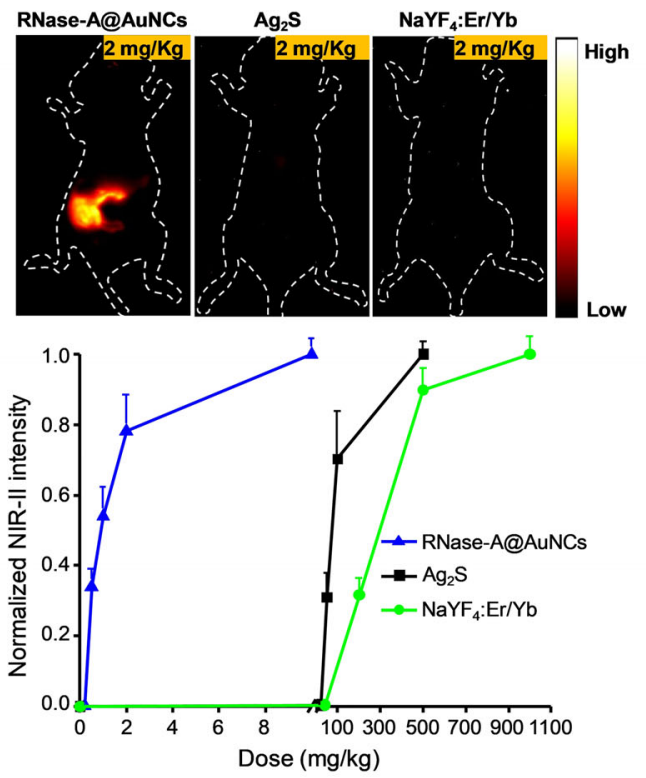

(B)

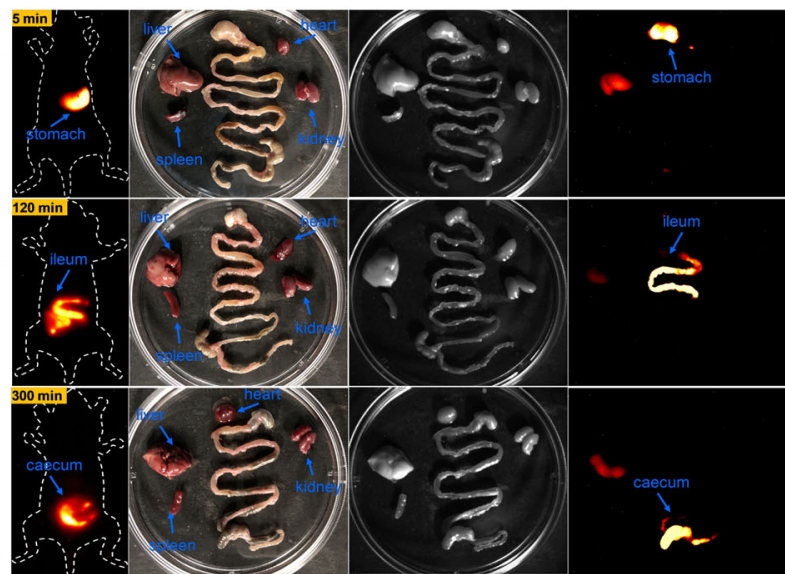

Figure 4. A) Signal-dose responses of NIR-II emitters in mice. The fluorescence was examined by InGaAs CCD camera (1000 nm LP, $200 \mathrm{~ms}, 808 \mathrm{~nm}$ excitation, $15 \mathrm{~mW} \mathrm{~cm}^{-2}$ ) $2 \mathrm{~h}$ after oral administration of emitters. The top panel shows representative NIR-II images of three nano-emitters in $\mathrm{Gl}$ tract $(n=3)$. Data are presented as mean values \pm SD. B) NIR-II imaging of gastrointestinal peristalsis by RNaseA@AuNCs. Mice were exposed to RNase-A@AuNCs by oral adminis tration at $2 \mathrm{mg} \mathrm{kg}^{-1}$ for real-time monitoring of gastrointestinal peristalsis under $808 \mathrm{~nm}$ excitation. indicated that AuNC based NIR-II imaging had a high resolution of $1.6 \mathrm{~mm}$ for GI tract, 4-fold higher than barium swallow (Figure S12).

Finally, we attempted to use RNase-A@AuNC for diagnosis of intestine cancer as the pathology changes may impede or block peristaltic movement, displaying signal changes in NIR-II imaging. At $2 \mathrm{~h}$ post oral administration, while RNase-A@AuNCs displayed consecutive distributions with uniform fluorescence in ileum of normal mice, dark craters were visualized at the forepart of ileum of intestine cancer mice, which were consistently present at the tumor nodules of intestine by ex vivo images as well as the H\&E staining sections with abnormities of glandular hyperplasia (Figure $5 \mathrm{~A}$ and Figure S13). AuNC based NIR-II imaging was able to clearly examine intestinal tumor nodules with diameters as small as $2.5 \mathrm{~mm}$, providing high imaging resolutions to abnormities in GI tract. The valley areas of ROI curves of all tested mice were calculated for statistical analysis. As shown in Figure 5D, tumor-burden mice displayed 11-fold increments of valley areas than healthy mice. These data indicated that NIR-II imaging of GI tract by RNase-A@AuNCs could well differentiate tumor-burden mice from the healthy.

(A)

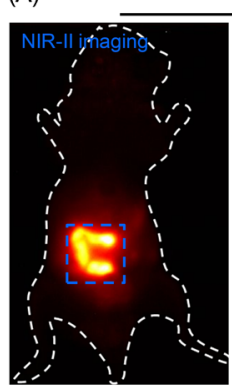

(B)

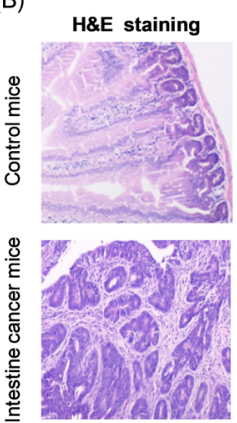

(C)
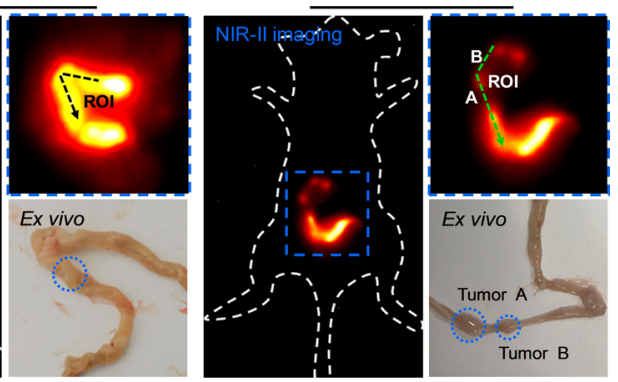

(D)
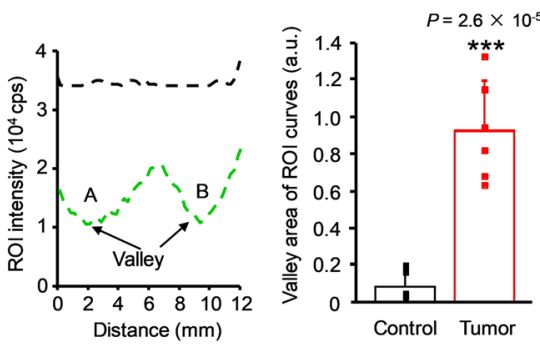

Figure 5. A) Mice with intestinal cancer and healthy mice were exposed to $2 \mathrm{mg} \mathrm{kg}^{-1}$ RNase-A@AuNCs by oral administration. After $2 \mathrm{~h}$, the NIR-II images were captured by InGaAs CCD camera. The dashed lines added on zoomed-in images represent the ROIs. The intensities of ROIs were measured by Image) 1.51. After NIR-II imaging, the animals were sacrificed to collect intestines for ex vivo examination. B) The animals were sacrificed to collect intestines for H\&E staining (magnification: $\times 400)$. C) The ROls were calculated based on the dashed lines of NIR-II images of healthy and tumor-burden mice by Image) 1.51. D) Valley areas of ROI-distance curves were measured by Origin Lab 8.0. $* * * p<0.001$ compared to control group by two tailed Student's $t$-test. 


\section{Conclusion}

In summary, red-shifted emissions of AuNCs at $1050 \mathrm{~nm}$ in NIR-II region could be achieved by engineering a unique protein corona on particle surface. The as-prepared RNaseA@AuNCs displayed a 1.9\% QY of NIR-II emissions, high stability and excellent biocompatibility. Compared to two reported NIR-II emitters $\left(\mathrm{Ag}_{2} \mathrm{~S}\right.$ and $\left.\mathrm{NaYF}_{4}: \mathrm{Er} / \mathrm{Yb}\right)$, RNaseA@AuNC exhibited > 50-fold higher sensitivity in GI tract. Notably, this emitter could be used to examine intestinal tumor nodules with diameters as small as $2.5 \mathrm{~mm}$. Overall, this study defined the first report of engineering protein corona on AuNCs for red shifts of emissions to NIR-II region and highlighted the promising utilities of RNase-A@AuNCs for diagnosis of intestinal tumors. Our findings provide new insights to engineer the surface coronas of nano-emitters for red-shifted emissions.

\section{Acknowledgements}

This work was supported by the National Natural Science Foundation of China (Number 21976126, 31671032), the grants from Key International Cooperation Projects of the Ministry of Science and Technology (2018YFE0120400), Key Project of social development of Jiangsu Province (BE2018653).

\section{Conflict of interest}

The authors declare no conflict of interest.

Keywords: fluorescent probes - gold nanoparticles . protein corona $\cdot$ red shift $\cdot$ tumor imaging

[1] M. Remedios, C. Campbell, D. M. Jones, P. Kerlin, Gastrointest Endosc. 2006, 63, 3-12.

[2] P. Wang, Y. Fan, L. Lu, L. Liu, L. Fan, M. Zhao, Y. Xie, C. Xu, F. Zhang, Nat. Commun. 2018, 9, 1-10.

[3] S. He, J. Song, J. Qu, Z. Cheng, Chem. Soc. Rev. 2018, 47, 4258 4278.

[4] X. D. Zhang, H. Wang, A. L. Antaris, L. Li, S. Diao, R. Ma, A. Nguyen, G. Hong, Z. Ma, J. Wang, Adv. Mater. 2016, 28, 6872 6879 .
[5] A. L. Antaris, H. Chen, K. Cheng, Y. Sun, G. Hong, C. Qu, S. Diao, Z. Deng, X. Hu, B. Zhang, Nat. Mater. 2016, 15, 235-242.

[6] S. Wang, Y. Fan, D. Li, C. Sun, Z. Lei, L. Lu, T. Wang, F. Zhang, Nat. Commun. 2019, 10, 1-11.

[7] a) A. Zebibula, N. Alifu, L. Xia, C. Sun, X. Yu, D. Xue, L. Liu, G. Li, J. Qian, Adv. Funct. Mater. 2018, 28, 1703451; b) Q. Wen, Y. Zhang, C. Li, S. Ling, X. Yang, G. Chen, Y. Yang, Q. Wang, Angew. Chem. Int. Ed. 2019, 58, 11001-11006; Angew. Chem. 2019, 131, 11117-11122.

[8] R. Li, Z. Ji, J. Dong, C. H. Chang, X. Wang, B. Sun, M. Wang, Y. P. Liao, J. I. Zink, A. E. Nel, ACS Nano 2015, 9, 3293-3306.

[9] Y. Nagai, Y. Tsutsumi, N. Nakashima, T. Fujigaya, J. Am. Chem. Soc. 2018, 140, $8544-8550$.

[10] S. Taghizadeh, V. Alimardani, P. L. Roudbali, Y. Ghasemi, E. Kaviani, Photodiagn. Photodyn. Ther. 2019, 25, 389-400.

[11] Y. Kong, J. Chen, H. Fang, G. Heath, Y. Wo, W. Wang, Y. Li, Y. Guo, S. D. Evans, S. Chen, Chem. Mater. 2016, 28, 3041-3050.

[12] G. Hong, J. T. Robinson, Y. Zhang, S. Diao, A. L. Antaris, Q. Wang, H. Dai, Angew. Chem. Int. Ed. 2012, 51, 9818-9821; Angew. Chem. 2012, 124, 9956-9959.

[13] R. Wang, L. Zhou, W. Wang, X. Li, F. Zhang, Nat. Commun. 2017, 8, 14702-14714.

[14] K. Pyo, V. D. Thanthirige, K. Kwak, P. Pandurangan, G. Ramakrishna, D. Lee, J. Am. Chem. Soc. 2015, 137, 8244-8250.

[15] a) C. Zhou, G. Hao, P. Thomas, J. Liu, M. Yu, S. Sun, O. K. Öz, X. Sun, J. Zheng, Angew. Chem. Int. Ed. 2012, 51, 10118-10122; Angew. Chem. 2012, 124, $10265-10269$; b) C. L. Liu, H. T. Wu, Y. H. Hsiao, C. W. Lai, C. W. Shih, Y. K. Peng, K. C. Tang, H. W. Chang, Y. C. Chien, J. K. Hsiao, Angew. Chem. Int. Ed. 2011, 50, 7056-7060; Angew. Chem. 2011, 123, 7194-7198.

[16] a) J. Lin, X. Zeng, Y. Xiao, L. Tang, J. Nong, Y. Liu, H. Zhou, B. Ding, F. Xu, H. Tong, Chem. Sci. 2019, 10, 1219-1226; b) Y. Zhang, G. Hong, Y. Zhang, G. Chen, F. Li, H. Dai, Q. Wang, ACS Nano 2012, 6, 3695-3702.

[17] a) Y. Chen, D. M. Montana, H. Wei, J. M. Cordero, M. Schneider, X. Le Guével, O. Chen, O. T. Bruns, M. G. Bawendi, Nano Lett. 2017, 17, 6330-6334; b) Y. Yu, Z. Luo, D. Chemvrier, D. Leong, P. Zhang, D. Jiang, J. Xie, J. Am. Chem. Soc. 2014, 136, $1246-1249$.

[18] J. Zheng, C. Zhou, M. Yu, J. Liu, Nanoscale 2012, 4, 4073-4083.

[19] I. L. Bergin, F. A. Witzmann, Int. J. Biomed. Nanosci. Nanotechnol. 2013, 3, 163-220.

[20] R. Würth, G. Hörmannsperger, J. Wilke, P. Foerst, D. Haller, U. Kulozik, J. Funct. Foods. 2015, 15, 116-125.

[21] X. Cai, X. Liu, J. Jiang, M. Gao, W. Wang, H. Zheng, S. Xu, R. Li, Small 2020, 16, 1907663.

Manuscript received: July 22, 2020

Accepted manuscript online: August 14, 2020

Version of record online: 


\section{Communications}

Fluorescent Probes

W. Wang, Y. Kong, J. Jiang, Q. Xie,

Y. Huang, G. Li, D. Wu, H. Zheng, M. Gao,

S. Xu, Y. Pan, W. Li, R. Ma, M. X. Wu, X. Li,

H. Zuilhof, X. Cai, R. Li*

पII-IIII

Engineering the Protein Corona Structure on Gold Nanoclusters Enables Red-

Shifted Emissions in the Second Nearinfrared Window for Gastrointestinal Imaging

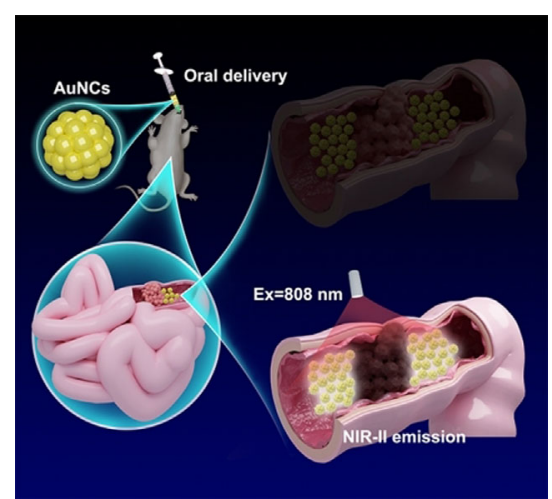

The precise control of the surface protein corona enabled the red-shifting of the emission of gold nanoclusters (AuNCs) into the NIR-II region. RNAse-A-encapsulated AuNCs allowed the visualization of the detailed structures of the Gl tract during gastrointestinal peristalsis, providing a non-invasive and $X$-ray-free technique to differentiate intestinal tumours. 